\title{
Dupriez, V. (2015). Peut-on réformer l'école? Approches organisationnelle et institutionnelle du changement pédagogique. Bruxelles : De Boeck.
}

\section{RECENSION}

L'ouvrage de Dupriez porte sur la question du changement pédagogique. Plus spécifiquement, l'auteur essaie de comprendre pourquoi les réformes pédagogiques sont si difficiles à conduire et mènent souvent à des échecs. Louvrage a aussi pour objectif de donner au lecteur des grilles de lecture théoriques, basées sur la recherche, pour comprendre le changement pédagogique.

Pour Dupriez, la réussite d'une réforme pédagogique passe généralement par sa capacité à susciter des innovations pédagogiques. Comprendre la différence entre réforme et innovation est donc essentiel: une réforme pédagogique vise à transformer les contenus, la manière dont ces contenus sont organisés ou l'organisation de l'enseignement et correspond à "une opération coordonnée de changement à large échelle " (p. 13). Par ailleurs, une innovation correspond à " une nouveauté dans un cadre particulier et pour des acteurs particuliers ». Ainsi, la thèse défendue par Dupriez est que «les systèmes éducatifs sont simultanément des lieux perméables aux innovations pédagogiques et par contre particulièrement hostiles aux réformes pédagogiques » (p. 38).

Cet ouvrage est divisé en trois parties. Dans la première, composée de trois chapitres, l'auteur s'appuie sur la sociologie des organisations pour présenter des modèles théoriques ainsi que des recherches menées en éducation qui permettent de penser le changement éducatif. Le premier chapitre porte sur les approches fonctionnalistes du changement, dans lequel Dupriez présente les tendances qui ont caractérisé la connaissance sur le fonctionnement des systèmes éducatifs depuis les années 1960 
jusqu'aux années 1990. Les recherches en éducation présentées dans ce chapitre ont en commun de partager une vision rationnelle du changement éducatif. Dans le deuxième chapitre, l'auteur présente deux approches, l'une basée sur la théorie de la contingence structurelle et dont il identifie deux logiques présentes dans les organisations éducatives : bureaucratique et professionnelle; et l'autre basée sur le concept de système faiblement articulé de Weick, servant à expliquer pourquoi les systèmes éducatifs sont hostiles aux réformes pédagogiques, mais perméables aux innovations pédagogiques. Dans le troisième chapitre, l'approche néo-institutionnaliste est présentée pour comprendre l'organisation scolaire comme "institution" traversée par des normes formelles autant que par des pressions normatives et des schèmes cognitifs partagés, mais qui est aussi à la recherche de légitimité.

Dans la deuxième partie, Dupriez présente, en trois chapitres également, de nouveaux modes de gouvernance des systèmes éducatifs, soit : la décentralisation et la gestion locale des établissements (chapitre quatre); la concurrence entre les écoles et les quasi-marchés scolaires (chapitre cinq); le pilotage par les résultats (chapitre six). Dans chacun de ces chapitres, l'auteur tente de rendre explicite la théorie du changement sous-jacente à ce mode de gouvernance; il fait également état de recherches qui ont étudié la mise en ouvre de telles politiques et qui permettent de discuter de leurs résultats.

Finalement, la troisième partie de l'ouvrage comporte deux chapitres. Dans le chapitre sept, Dupriez présente des initiatives de réformes qui reposent sur la conviction qu'un accroissement de la logique de standardisation serait la meilleure stratégie possible pour améliorer la qualité du service éducatif; dans le chapitre huit, il expose le point de vue opposé, expliquant de quelle manière ces pratiques amènent à une prolétarisation du métier d'enseignant, proposant un plaidoyer en faveur de la reconnaissance de l'importance du développement professionnel des enseignants.

Au final, l'ouvrage de Dupriez suit une logique fort bien articulée. Ainsi, des grilles de lecture théorique pour comprendre le changement éducatif sont offertes en début d'ouvrage, qu'il exploite ensuite dans la présentation des modes de gouvernance qui coexistent actuellement dans les systèmes éducatifs de la plupart des pays occidentaux. Par exemple, dans la deuxième partie du livre, l'auteur expose les politiques de gouvernance qu'il explicite ensuite par la théorie du changement qu'elles mobilisent pour, enfin, présenter des recherches relatives à la mise en œuvre de ces politiques et à certains de leurs effets sur la performance des élèves. De plus, les trois formes de gestion locale sont très bien expliquées, par des exemples concrets contextuels tirés de différents pays. À cet égard, Dupriez présente une analyse quasi comparative entre différents États, ce qui la rend d'autant plus intéressante.

Une autre force de l'ouvrage est son cadre théorique, qui permet, d'une part, de bien saisir les tendances des recherches en ce domaine; d'autre part, de comprendre l'évolution du changement éducatif. Cela dit, si Dupriez voulait donner au lecteur des grilles de lectures théoriques pour comprendre le changement éducatif, celles-ci restent, sur certains aspects, limitées, car Dupriez ne place pas les approches théoriques utilisées dans le cadre plus large de la sociologie des organisations : il y a par exemple seulement une exposition rapide de l'école de la contingence structurelle et, dans le chapitre suivant, de son opposition avec l'approche néo-institutionnaliste. Or, il aurait été pertinent de présenter et situer les différentes approches dans un cadre plus large en termes historiques et paradigmatiques. Également, certains concepts provenant de ces approches sont peu exploités. Par exemple, en ce qui a trait à l'approche néo-institutionnaliste, le concept d'isomorphisme, passé rapidement en revue par l'auteur, aurait pu expliquer les évolutions semblables dans les politiques et modes de gouvernance que l'on trouve dans 


\section{CHRONIQLEE}

différents contextes. Par ailleurs, les recherches exposées dans le premier chapitre de l'ouvrage sont présentées très vite par l'auteur, leurs effets, ainsi que la dynamique entre le processus réformiste et les différentes institutions concernées est difficilement repérable à cause notamment d'un manque d'exemples concrets et mieux contextualisés du changement (contextualisation et exemplification qu'il exploitera très bien cependant dans la deuxième partie pour expliquer les modes de gouvernance). Cela dit, il faut dire que dans un ouvrage très court (160 pages), Dupriez réalise une analyse très pertinente du changement éducatif en exploitant de manière efficace les ressources théoriques et les résultats de la recherche scientifique.

Finalement, force est d'admettre qu'il s'agit d'un livre excellent et extrêmement pertinent pour ceux qui souhaitent comprendre les théories qui expliquent le changement éducatif et l'état de la recherche en ce domaine. De plus, l'approche globale et presque comparative de l'ouvrage permet de bien saisir les problématiques communes à la plupart des pays occidentaux. Un ouvrage que les décideurs politiques, les chercheurs en éducation et toutes personnes qui s’intéressent aux réformes éducatives, à leurs effets et aux stratégies de changement pédagogique, devraient lire et sur lequel ils pourraient méditer.

\section{Pour citer cet article}

Morales Perlaza, A. (2016). Dupriez, V. (2015). Peut-on réformer l'école? Approches organisationnelle et institutionnelle du changement pédagogique. Bruxelles: De Boeck. Formation et profession 24(2), 90-92.

http://dx.doi.org/10.18162/fp.2016.a101 